\title{
Benajir Wolf: Sinnverstehende Psychomotoriktherapie mit Erwachsenen
}

Ernst Reinhardt Verlag, 2019, München, 142 Seiten, 29,90€(D)

E in Titel, der neugierig stimmt und Interessensfragen aufwirft. Wo verortet sich die sinnverstehende Psychomotoriktherapie? Ist es eine erweiterte Form der Psychomotorik? Ermöglicht die angekündigte Therapieform, die Verknüpfungspunkte zwischen Körpertherapie und Körperpsychotherapie näher zu definieren? Wird den TherapeutInnen in den Grenzbereichen auf diese Weise mehr Sicherheit in der therapeutischen Begleitung gegeben?

In der Kurzzusammenfassung, auf der Rückseite des Buches, wird mit folgenden Worten für die Methode geworben: „Mit der sinnverstehenden Psychomotoriktherapie liegt erstmals eine psychoanalytisch fundierte Therapiemethode für die psychomotorische Arbeit mit Erwachsenen vor." Es wird beschrieben, dass die Methode sowohl durch den verstehenden Ansatz nach Seewald als auch durch psychoanalytische Konzepte der Körperpsychotherapie beeinflusst ist.

Das Buch selbst ist in zwei Teile gegliedert. Im ersten Teil wird die Entstehung der sinnverstehenden Psychomotoriktherapie (PMT) sowie die theoretischen Grundlagen beschrieben, auf welchen der Ansatz aufbaut. Auch findet hier eine Verortung sowie Abgrenzung des Ansatzes im Feld verschiedener bestehender Therapiemethoden statt.

Der zweite Teil umfasst das Konzept und die Methode der PMT. Hier geht es besonders um den Begriff des Verstehens bzw. Nicht-Verstehens sowie die darauf aufbauende sinnverstehende Haltung und Methode. Die Autorin setzt sich weiter mit den Themen Menschen- und Körperbild, persönliche Grundhaltung und therapeutische Haltung und Handeln, mit der Bedeutung der therapeutischen Beziehung sowie den Körperbedeutungen und möglichen Arbeitswei- sen auseinander. Anschließend wird das Arbeitssetting und die Arbeit mit verschiedenen Patientengruppen (unterteilt in die Ent-

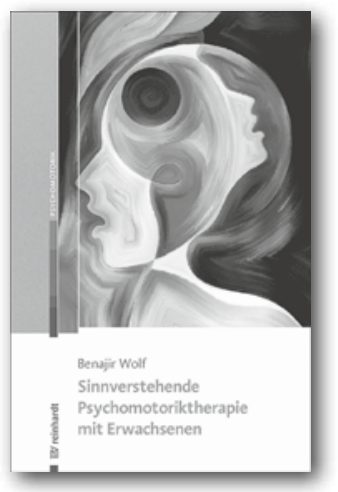
wicklungsphasen nach Freud und Erikson) beschrieben. Den Abschluss des Buches bildet ein Fazit mit Ausblick.

Zielgruppe des Buches sind bereits praktizierende, aber auch in Ausbildung oder Studium befindliche TherapeutInnen, PsychomotorikerInnen und MotologInnen. Betont wird jedoch, dass das Buch letztendlich für PatientInnen geschrieben ist.

Verstanden habe ich für mich, dass es in dieser Methode in erster Linie um den Versuch des Verstehens geht bzw. darum, die PatientInnen in ihrem eigenen Verstehensprozess zu begleiten: eine Begleitung von Verunsicherung und Orientierungslosigkeit im Erkrankungsspektrum oder bestehenden Lebensthemen hin zu mehr Sicherheit, Orientierung und Klarheit.

Das Buch punktet durch seinen klaren Aufbau, eine gute Struktur und hilfreiche Zusammenfassungen an einigen Kapitelenden. Deutlich wird die stark analytisch geprägte Grundhaltung, welche sich durch alle Kapitel zieht. Der Sprachstil sowie Tiefgang in den Themen schwankt jedoch bezüglich der verschiedenen Zielgruppen des Buches. So werden in manchen Kapiteln Basics vermittelt, welche PatientInnen einen Einblick in die therapeutischen Grundlagentheorien bieten können, in anderen Kapiteln und Abschnitten zeigt sich jedoch der Bedarf nach einem gewissen Grundfachwissen im therapeutischen Spektrum. Mit Blick auf die als Zielgruppe des Buches be- 
schriebenen Patientlnnen entstanden bei mir doch einige Irritationen. Im ersten Teil des Buches findet neben der Beschreibung der Grundlagen und Hintergrundtheorien der PMT eine klare Abgrenzung zu Haltungen und Arbeitsweisen anderer therapeutischer Fachrichtungen statt. Die Irritation entstand durch die wertende bis abwertende Beschreibung anderer therapeutischer Fachrichtungen. Diese erscheint mir in ihrer Art eher oberflächlich und mit Vorurteilen gespickt zu sein. Hierbei frage ich mich, was PatientInnen, aber auch Auszubildende und StudentInnen mit dieser Beschreibung anfangen sollen. Kann so eine Basis in der Beziehung zu TherapeutInnen anderer als analytisch geprägter Therapierichtungen und deren Methodenumsetzung be- bzw. entstehen? Meine Motivation, ein Fachbuch zu lesen, ist, eine Methode oder Theorien kennenzulernen, mein Spektrum zu erweitern und Neues zu lernen. Eine Abgrenzung scheint mir in manchen Momenten sinnvoll, um Unterschiede und Gemeinsamkeiten verschiedener Therapiemethoden zu verstehen und einzuordnen. Andere Methoden herabzustufen, um eigene hervorzuheben, empfinde ich als unnötig. Es werden Gefahren im therapeutischen Setting und Interaktionsrahmen angesprochen, Lösungswege erkenne ich vereinzelt. Als eine Gefahr wird beschrieben, dass Therapeutlnnen sich durch die Verwendung verschiedener bzw. neuer, manchmal fachfremder Interventionen an den PatientInnen ausagieren würden.

Im zweiten Teil des Buches werden Methoden als Teil der PMT beschrieben, welche anderen therapeutischen Ausbildungen und Ansätzen, wie zum Beispiel der Systemischen und auch der Drama- und Theatertherapie, zuzuordnen sind. Dies widerspricht meiner Meinung nach den vorherigen Aussagen. Die fachliche Auseinandersetzung mit dem Menschen- und Körperbild, der persönlichen Grundhaltung und der therapeutischen Haltung wirkt eher wie eine generelle Erklärung der Begriffe mit Bezug auf das therapeutische Setting, wie sie auch in den allgemeinen therapeutischen Standardwerken zu finden ist. Die Absätze sind schlüssig beschrieben, jedoch wird die Verortung der PMT für mich nicht ganz klar. Deutlicher wird das Arbeiten der PMT in der Beschreibung des Settings und der Zielgruppe. Hier werden anhand von Therapiesituationen beispielhaft Interventionen und Handlungen beschrieben.

Die PMT hat es in meinen Augen nicht nötig, sich auf diese Art zu präsentieren, zeigt sich die Methode selbst doch als schlüssig aufgebaut und fachlich untermauert.

\section{Maren Meinhold}

DOI 10.2378/ ktb2022.arto5d 